\title{
Transcriptional Regulation of Tal2 Gene by All-trans Retinoic Acid (atRA) in P19 Cells
}

\author{
Takanobu Kobayashi, ${ }^{a}$ Masayo Suzuki, ${ }^{a}$ Masayuki Morikawa, ${ }^{a}$ Katsuhito Kino, ${ }^{a}$ \\ Sei-ichi Tanuma, ${ }^{b}$ and Hiroshi Miyazawa, ${ }^{* a}$ \\ ${ }^{a}$ Kagawa School of Pharmaceutical Sciences, Tokushima Bunri University; 1314-1 Shido, Sanuki, Kagawa 769-2193, \\ Japan: and ${ }^{b}$ Department of Biochemistry, Faculty of Pharmaceutical Sciences, Tokyo University of Science; 2641 \\ Yamazaki, Noda, Chiba 278-8510, Japan. \\ Received August 28, 2014; accepted November 24, 2014; advance publication released online December 5, 2014
}

TAL2 is a transcription factor required in the normal development of mouse brain. In a previous study, we demonstrated that the expression of Tal2 gene is induced by the complex of all-trans retinoic acid (atRA) and retinoic acid receptor $\alpha(\operatorname{RAR} \alpha)$ in mouse embryonal carcinoma P19 cells. atRA is also known to be important in inducing P19 cells to differentiate into the neural lineage. Therefore, we believe that the function of TAL2 in neural differentiation may be clarified by utilizing P19 cells. As the atRA-RAR $\alpha$ complex induced the expression of Tal2, we focused on the regulatory region that is involved in its transcription. The atRA-RAR $\alpha$ complex occupies a characteristic retinoic acid response element (RARE) located in the promoter of target genes. Therefore, we searched for RARE on the mouse Tal2 and found that a RARE-like element was located in the intron. We also found that a TATA-box-like element was located in the 5'-region of Tal2. Involvement between transcriptional activity and the TATA-box-like element was confirmed in the luciferase assay, and TATA-box binding protein was bound to this element upstream of Tal2 in P19 cells. atRA signaling activated the transcription through the RARE-like element, and RAR $\alpha$ was bound to this element on Tal2 in P19 cells. In addition, the interaction between these elements on Tal2 was shown in the chromatin immunoprecipitation assay. These results suggest that the transcription of Tal2 is coordinately mediated by two distal regulatory elements.

Key words Tal2 gene; retinoic acid receptor $\alpha(\mathrm{RAR} \alpha)$; TATA-box binding protein (TBP); retinoic acid response element (RARE); TATA-box

TAL2 $2^{1,2)}$ is a transcription factor required for the normal development of mouse brain. The expression of Tal2 gene is observed in the diencephalon, mesencephalon, and metencephalon of the developing mouse brain. ${ }^{3)}$ Tal2-null mutant mice are viable at birth and initially appear normal. However, they develop signs of runting and die between 13 and $32 \mathrm{~d}$ after birth. ${ }^{4}$ Therefore, Tal2 is thought to play a pivotal role in development of the brain.

We found that Tal2 expression is altered in P19 cells after addition of all-trans retinoic acid (atRA) and suspension culture for cell aggregation. Moreover, we showed that its expression is induced by atRA. ${ }^{5)}$ P19 cells are a line of pluripotent embryonal carcinoma and appear to differentiate into derivatives of three germ layers-endoderm, mesoderm, or ectoderm-depending on the inducers and culture conditions, using the same mechanisms as normal embryonic cells. atRA treatment and cell aggregation have an important role in the induction of neural differentiation in P19 cells. ${ }^{6-8)}$ Because these cells have been used as a model in studies of neural differentiation, we believe that the function of Tal2 in development may be clarified by utilizing P19 cells.

atRA, which is a metabolic product of vitamin $\mathrm{A}$, is one of the most important morphogens, and is a signal molecule involved in neural differentiation. ${ }^{9-11)}$ atRA is also known to be capable of inducing embryonic stem cells and embryonal carcinoma cells to differentiate into neural lineages. ${ }^{12,13)}$ atRA functions as the activating ligand for retinoic acid receptors (RARs), and atRA-RAR complexes regulate the expression of over 500 target genes as a transcription factor. ${ }^{11,13-16)}$ RARs are a member of the nuclear receptor superfamily, ${ }^{17,18)}$ and consist of three isotypes: $\alpha, \beta$, and $\gamma$. In response to atRA signaling, RARs occupy characteristic retinoic acid response elements (RAREs) located in the promoter regions of target genes. RAREs are classically described as direct repeats of the hexameric motif $(A / G) G(G / T) T C A$ separated by 1, 2, or 5 nucleotides (referred to as DR1, DR2, or DR5, respectively). ${ }^{19-21)}$ Recently, the recurrent motif $(\mathrm{A} / \mathrm{G}) \mathrm{G}(\mathrm{G} / \mathrm{T}) \mathrm{T}(\mathrm{G} / \mathrm{C}) \mathrm{A}$, which differs from the classical consensus motif $(A / G) G(G / T) T C A$ at position 5, with a $\mathrm{G}$ instead of a $\mathrm{C}$, was reported from the alignment of several RAREs. ${ }^{20}$

In a previous study, we also showed that RAR $\alpha$ was involved in the induction of Tal2 in P19 cells after the addition of atRA and suspension culture for cell aggregation. As the atRA-RAR $\alpha$ complex induced the expression of Tal2, we focused on the regulatory region involved in its transcription. Therefore, we searched for a RARE motif on Tal2 and found a RARE-like element in the intron of Tal2. Moreover, a TATAbox-like element, which might participate in transcription as core promoter recognized by basal transcriptional factors, was mapped in the 5'-region of Tal2. Subsequently, we investigated the relationship between the transcriptional activities and these elements, and the association between these elements and bound proteins. In addition, we examined the interaction between these elements on Tal2 in P19 cells.

\section{MATERIALS AND METHODS}

Cell Culture and Neural Differentiation P19C6, a sub- 
clone of the P19 mouse embryonic carcinoma cell line, was used in this study. ${ }^{22)}$ P19C6 was provided by the RIKEN BRC through the National Bio-Resource Project of the MEXT, Japan. P19 cells were cultured in $\alpha$-MEM (Sigma-Aldrich, St. Louis, MO, U.S.A.) supplemented with $10 \%$ fetal bovine serum (FBS) (Life Technologies, Carlsbad, CA, U.S.A.) and $2 \mathrm{~mm}$ L-glutamine (Kanto Chemical, Tokyo, Japan). To induce neural differentitation, cells were aggregated in a suspension culture dish (SUMILON, Tokyo, Japan) at a seeding density of $2 \times 10^{5}$ cells $/ \mathrm{mL}$ in the presence of $1 \mu \mathrm{M}$ atRA (Sigma-Aldrich) dissolved in dimethyl sulfoxide (DMSO) (Sigma-Aldrich). DMSO concentration in culture condition was $0.01 \%$.

5'-Rapid Amplification of cDNA Ends (RACE) of Tal2 RNeasy Mini Kits (QIAGEN, Hilden, Germany) were used to isolate total RNA from P19 cells according to the manufacturer's instructions. To identify the 5'-ends of Tal2, the SMARTer $^{\mathrm{TM}}$ RACE cDNA Amplification Kit (TaKaRa Bio, Shiga, Japan) was used according to the manufacturer's instructions. The $5^{\prime}$-end of Tal2 was amplified by Ex taq (TaKaRa) and a mouse Tal2 gene-specific primer, 5'-AGA GGC TCA ACT GAA GTC GAA-3'. Subsequently, polymerase chain reaction (PCR) products were cloned into the pGEM-T Easy vector (Promega, Madison, WI, U.S.A.) for sequencing.

Western Blotting P19 cells were treated with atRA for $0,3,6,12$ and $24 \mathrm{~h}$ in suspension culture. Treated cells were washed with phosphate buffered saline (PBS) and then harvested by centrifugation. Samples were resuspended in lysis buffer $(62.5 \mathrm{~mm}$ Tris- $\mathrm{HCl}$ [pH 6.8], 10\% glycerol, 2\% sodium dodecyl sulfate (SDS), $0.05 \mathrm{mg} / \mathrm{mL}$ bromophenol blue) and boiled for $5 \mathrm{~min}$. They were then separated by SDS-polyacrylamide gel electrophoresis (PAGE) and transferred to an Immobilon-P transfer membrane (Merck Millipore, Darmstadt, Germany) in transfer buffer (25 mM Tris, $192 \mathrm{~mm}$ glycine, $10 \%$ methanol) using the Trans-Blot Turbo Transfer System (BioRad, Hercules, CA, U.S.A.). After blocking with 5\% skim milk in $0.1 \%$ TBS-T (10 mm Tris- $\mathrm{HCl}$ [pH 7.4], $150 \mathrm{~mm} \mathrm{NaCl}$, $0.1 \%$ Tween 20 ) for $1 \mathrm{~h}$ at room temperature, membranes were incubated with anti-RAR $\alpha$ antibody, anti-TFIID (TBP) antibody (Santa Cruz Biotechnology, Santa Cruz, CA, U.S.A.), and anti- $\beta$-Actin antibody (MEDICAL \& BIOLOGICAL LABORATORIES, Nagoya, Japan). Subsequently, membranes were incubated with secondary antibody conjugated to horseradish peroxidase (Cell Signaling Technology, Danvers, MA, U.S.A.), and were detected with the Immobilon Western chemiluminescent horse-radish peroxidase (HRP) substrate.

Luciferase Reporter Assay The 5'-region of Tal2 was amplified from mouse genomic DNA, and was cloned into the firefly luciferase reporter plasmid, pGL4.10[luc2] vector (Promega). P19 cells $\left(8 \times 10^{5}\right.$ cells) were seeded onto 12 -well plates and transfected with vectors using Lipofectamine 2000 (Life Technologies) according to the manufacturer's instructions. Medium was replaced at $5 \mathrm{~h}$ after transfection with atRA or DMSO in suspension culture. ${ }^{23)}$ After a further $20 \mathrm{~h}$, luciferase activity was assayed using the Dual-Glo Luciferase Assay System (Promega) according to the manufacturer's instructions. Subsequently, the reporter vector containing the Tal2 gene region from -240 to +73 was used to analyze the TATA-box-like element. For this purpose, a reporter vector lacking this element (-240 $\Delta$ TATA) was also constructed. Luciferase activity was performed in the same manner as for the 5'-region of Tal2.
For analysis of the TATA-box-like element upstream of Tal2, we utilized the CheckMate/Flexi Vector Mammalian Two-Hybrid System (Promega). The firefly luciferase reporter plasmid pGL4.31[luc2P/GAL4UAS/Hygro] vector was digested with NheI and HindIII to remove the adenovirus major late promoter. The following oligonucleotides were inserted into pGL4.31 digested with NheI and HindIII: TATA(Tal2), 5'-GC T AGC GGC GGT GTC CTATAAAG GCT GTG GCA GAG AC CCTGCGT CCA GGC GAGGGC GCA CAA AGC TT-3'; and

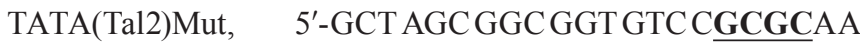
G GCT GTG GCA GAG ACC CTG CGT CCA GGC GAG GG CGCACAA AGC TT-3'. Mutated bases are shown in boldface. In cotransfection experiments with MyoD fused to VP16 expression vector, Id fused to GAL4 expression vector and reporter vector, $\mathrm{P} 19$ cells $\left(4 \times 10^{5}\right.$ cells) were seeded onto 24 -well plates and were transfected with these vectors using Lipofectamine 2000 (Life Technologies) according to the manufacturer's instructions. Luciferase activity was assayed by DualGlo Luciferase Assay System at $24 \mathrm{~h}$ after cotransfection.

For analysis of the RARE-like element in the intron of Tal2, RAR $\alpha$ fused to VP16 (VP16-RARA) was cloned into the pCAGGS vector. ${ }^{24)}$ Moreover, to remove GAL4-binding sites, the pGL4.31 vector was digested with KpnI and NheI, and the following oligonucleotides were inserted into pGL4.31 digested with KpnI and NheI: DR5(Tal2)×3, 5'-GGT ACC TGA ACT TTG ACT CAC CTG TGA ACT TTG ACT CAC CTG TGA ACT TTGACTCACCTGGCA GC-3'; and DR5(Tal2)Mut $\times 3$, 5'-GGT ACC AAA ACT TTG ACT CAC CTG AAA ACT TTG ACT CAC CTG AAA ACT TTG ACT CAC CTG GCT AGC-3'. The vector contained three tandem repeats of the RARElike element (underlined). Mutated bases in DR5(Tal2)Mut are shown in boldface. The luciferase activities in P19 cells transfected with both the VP16-RARA expression vector and the reporter vector were measured in the same manner as cotransfection experiments with the TATA-box-like element. Luciferase activities from the RARE-like element with atRA signaling were measured in P19 cells.

All experiments were carried out in triplicate, and firefly luciferase activity was normalized using Renilla luciferase activity. Statistical significance was determined by $t$ test $(* p<0.05)$.

Preparation of Nuclear Proteins and Electrophoretic Mobility Shift Assay (EMSA) Nuclear extracts were prepared as described previously. ${ }^{16)}$ Briefly, P19 cells $\left(1 \times 10^{7}\right.$ cells), which were treated with $1 \mu \mathrm{m}$ atRA for 0 and $3 \mathrm{~h}$ in suspension culture, were harvested by centrifugation. The cell pellet was resuspended in buffer A (10 mM $N$-(2-hydroxyethyl)piperazine- $N$ '-2-ethanesulfonic acid (HEPES)-KOH $[\mathrm{pH}$ 7.9], $10 \mathrm{~mm} \mathrm{KCl}, 1.5 \mathrm{~mm} \mathrm{MgCl}_{2}, 0.5 \mathrm{~mm}$ dithiothreitol (DTT), Complete Mini ethylenediaminetetraacetic acid (EDTA)-free protease inhibitors [Roche Diagnostics, Basel, Switzerland]) and incubated on ice for $10 \mathrm{~min}$. Nuclei were collected from the lysates by centrifugation at $1000 \times \boldsymbol{g}$ for $5 \mathrm{~min}$. Pellets were then resuspended in buffer $\mathrm{C}$ (20 mM HEPES [pH 7.9], $420 \mathrm{~mm}$ $\mathrm{NaCl}, 1.5 \mathrm{~mm} \mathrm{MgCl}_{2}, 0.2 \mathrm{~mm}$ EDTA, 25\% glycerol, $0.5 \mathrm{~mm}$ DTT, Complete Mini EDTA-free protease inhibitors [Roche]) and incubated on ice for $30 \mathrm{~min}$. Supernatants were collected by centrifugation at $20000 \times \boldsymbol{g}$ for $2 \mathrm{~min}$. The protein concentration of nuclear extracts was measured using the Bio-Rad Protein Assay Dye Reagent Concentrate (Bio-Rad).

EMSA was also performed as described previously. ${ }^{16)}$ 
A

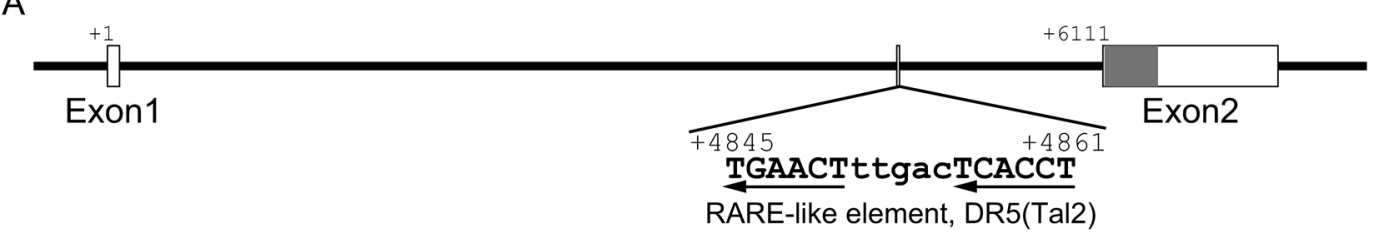

B .....cccetcctgecgccagacaaagggeggtgtcctataaaggetgtggcagagaccetg TATA-box-like element, TATA(Tal2)

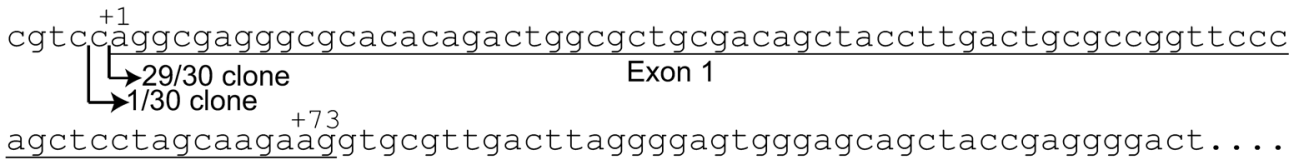

C

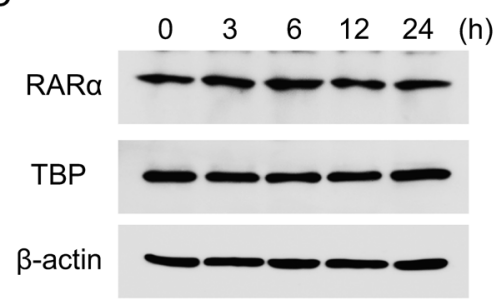

Fig. 1. Regulatory Region of Mouse Tal2

(A) Map of putative RARE, the RARE-like element "DR5(Ta12)," on Tal2. Arrow under the sequence indicates the hexameric motif of a direct repeat. This site is separated by 5 nucleotides. Exons are indicated by open box. Gray box shows protein-coding region (B) Sequence of 5'-region of Tal2. 5'-end of Tal2 (arrows) in P19 cells determined by 5 '-RACE. Putative TATA-box, TATA-box-like element "TATA(Tal2)" (bold face and underlined) is located 30 bp upstream of the 5 '-end. Exon 1 is indicated by the underlined sequences. (C) P19 cells were treated with atRA in suspension culture for 0, 3, 6, 12 or $24 \mathrm{~h}$. Expression of RAR $\alpha$ and TBP in these cells was detected by Western blotting. $\beta$-Actin was used as a loading control.

Briefly, Alexa 680-labeled probes containing following sequences were used for EMSA: TATA(Ta12), 5'-Alexa680GCGGTGTCCTATAAA GGCTGTGGCA-3'; TATA(Tal2)Mut, 5'-Alexa680-GCG GTG TCC GCGCAAG GCT GTG GCA-3'; DR5(Tal2), 5'-Alexa680-CTA GGG GTG AAC TTT GAC TCA CCT GCC AGT G-3'; and DR5(Tal2)Mut， 5'-Alexa680-CTA GGG GAA AAC TTT GAC TCA CCT GCC AGT G-3'. Mutated bases are shown in boldface. Nuclear extracts $(10 \mu \mathrm{g})$ were incubated for $10 \mathrm{~min}$ on ice in reaction buffer $(10 \mathrm{~mm}$ Tris$\mathrm{HCl}$ [pH 7.5], $50 \mathrm{~mm} \mathrm{NaCl}, 0.5 \mathrm{~mm}$ EDTA, $0.5 \mathrm{~mm}$ DTT, $1 \mathrm{~mm}$ $\mathrm{MgCl}_{2}$, and $2 \mathrm{mg}$ poly $\left.(\mathrm{d} I-\mathrm{d} C) \cdot \operatorname{poly}(\mathrm{d} I-\mathrm{d} C)\right)$. Alexa 680-labeled probe was then added to nuclear extracts. These mixtures were incubated for $30 \mathrm{~min}$ at room temperature. Samples were separated by electrophoresis on 5\% native PAGE. Gels were analyzed using the Odyssey Infrared Imaging System (LI-COR Biosciences, Lincoln, NE, U.S.A.). For supershift analysis, anti-RAR $\alpha$ antibody (ActiveMotif, Carlsbad, CA, U.S.A.) or anti-TFIID (TBP) antibody (Santa Cruz Biotechnology) was added to nuclear extract before addition of the probe, and then incubated at room temperature for $1 \mathrm{~h}$.

Chromatin Immunoprecipitation (ChIP) Assay ChIP assay was performed by SimpleChIP Plus Enzymatic Chromatin IP Kit (Magnetic Beads) (Cell Signaling Technology) according to the manufacturer's instructions. This assay was used for P19 cells $\left(4 \times 10^{6}\right.$ cells for the RARE-like element or $2 \times 10^{5}$ cells for the TATA-box-like element) treated with atRA for $3 \mathrm{~h}$ in suspension culture. The isolated chromatin DNA was subjected to PCR analysis using AmpliTaq Gold 360 Master Mix (Life Technologies). The following gene specific primers were used to amplify: TATA(Tal2), 5'-TTC TTC CTC CAC TGC TCC TTG-3' (sense) and 5'-TCT
CTG CCA CAG CCT TTA TAG GAC A-3' (antisense); and DR5(Tal2) 5'-CACCAA GGA ACA GCA AGT GGA-3' (sense) and 5'-GGCCTT TCC ATA GCT GACCTT-3' (antisense); negative control, 5'-TGA GCC ACC ATA TGGATGCTG-3' (sense) and 5'-CAACTGCAGCTGTTA GCT GTG-3' (antisense).

\section{RESULTS AND DISCUSSION}

Regulatory Region of Tal2 Gene atRA signaling regulates the transcription of target genes through the binding of RARs to RARE, and is involved in the induction of Tal2 in P19 cells. ${ }^{5)}$ Thus, we aimed to predict the regulatory region of Tal2, and searched for the RARE motif located within $\pm 10 \mathrm{~kb}$ from both the transcription start site (TSS) and the end of Tal2, with reference to previous studies. ${ }^{20)}$ Mouse Tal2 consists of two exons and an intron. Although RARE has not been mapped in the 5'- or 3'-flanking regions of Tal2, we found a RARE-like motif, which has been reported, ${ }^{20)}$ in the intron of Tal2. This motif was designated "DR5(Tal2)" in this paper. The direct repeat of the hexameric motif at this site is separated by 5 nucleotides (Fig. 1A). This raises the question of whether the TSS of Tal2 is located upstream of the first exon. Therefore, we mapped the 5'-end of Tal2 in P19 cells by $5^{\prime}$-RACE. Thirty clones were sequenced and aligned to the genome (Fig. 1B). Among these clones, 29 had the same position as the $5^{\prime}$-end of Tal2, $4 \mathrm{bp}$ upstream of the TSS reported previously. ${ }^{25)}$ Moreover, the TATA-box-like element (bold face in Fig. 1B), which was designated "TATA(Tal2)" in this paper, was predicted to be located $30 \mathrm{bp}$ upstream of the TSS that we identified. The TATA-box is a core promoter motif and has a consensus sequence of $\operatorname{TATA}(\mathrm{A} / \mathrm{T}) \mathrm{A}(\mathrm{A} / \mathrm{T})(\mathrm{A} / \mathrm{G})$, where the 
A

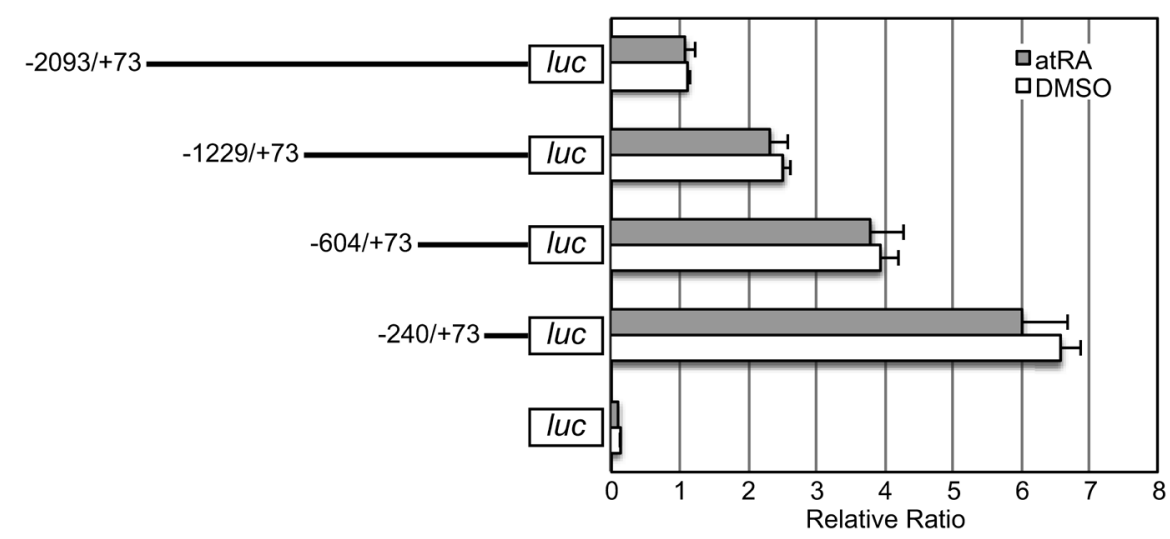

B

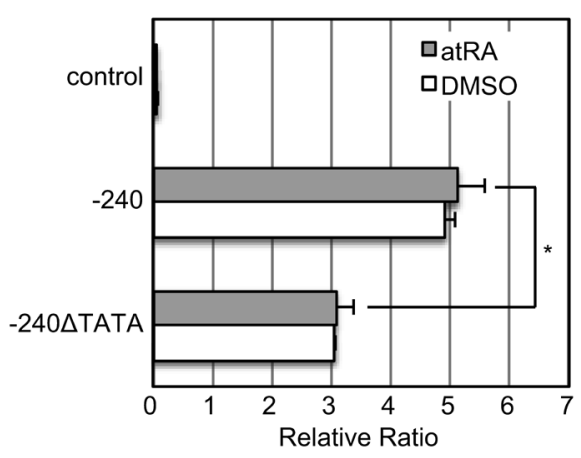

C

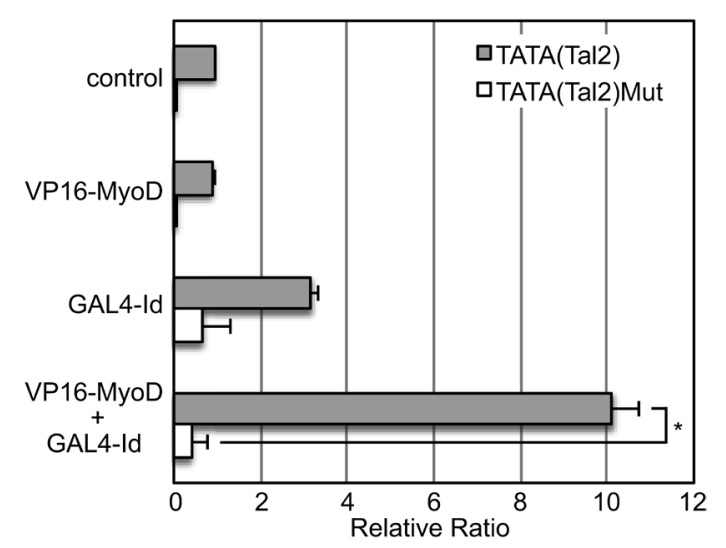

Fig. 2. Transcriptional Activity of TATA(Tal2) in P19 Cells

(A) Transcriptional activity of 5'-region on Tal2 in P19 cells. Deletion constructs are shown on the left. P19 cells were transfected with these constructs, followed by the treatment of atRA or DMSO in suspension culture, and then luciferase activity was observed. Data represent means \pm S.E. of three independent experiments. (B) Reporter vector containing 5 '-region from -240 to +73 of Tal2 was used for analysis of TATA(Tal2) transcriptional activity. Deletion of TATA(Tal2) decreased luciferase activity in P19 cells. Control indicates the luciferase activity of reporter vector without the $5^{\prime}$-region. Data represent means \pm S.E. of three independent experiments. $* p<0.05$. $(C)$ The two-hybrid system adapted for use in mammalian cells was utilized to analyze the transcriptional activity of TATA(Ta12) or TATA(Tal2)Mut. VP16-MyoD and GAL4Id indicate MyoD fused to the herpes simplex virus VP16 activation domain and yeast GAL4 DNA-binding domain, respectively. Luciferase activity from TATA(Tal2) or TATA(Ta12)Mut was measured in P19 cells that were cotransfected with these vectors. Controls were cotransfected with empty expression vectors. Data represent means \pm S.E. of three independent experiments. $* p<0.05$.

upstream $\mathrm{T}$ nucleotide is most commonly at -31 or -30 relative to $\mathrm{TSS}^{26-28)}$

We assumed that TATA(Tal2) in 5'-region and DR5(Tal2) in the intron were involved in the transcription of Tal2. It is known that TATA-box binding protein (TBP) binds to the TATA-box, and that $\operatorname{RAR} \alpha$, which is involved in the induction of Tal2, binds to RARE. Thus, we examined the expression of these proteins in P19 cells with atRA treatment in suspension culture (Fig. 1C). As a result, it was observed that these proteins remained unchanged for $24 \mathrm{~h}$ after atRA treatment.

Relationship between TATA(Tal2) and Transcription of Tal2 We constructed reporter vectors containing the 5'-region of Tal2 and examined the transcriptional activity of these regions in P19 cells (Fig. 2A). Luciferase activity increased in P19 cells transfected with these constructs, as compared with empty vector. However, their activity with atRA treatment was almost the same with DMSO treatment. Under adhesion conditions, luciferase activity was observed in P19 cells transfected with these reporter vectors, similar to suspension culture (data not shown). TATA(Ta12) was contained in these vectors and might be involved in the transcription. The TATAbox is the predominant DNA element of core promoters that directs transcriptional initiation, and is recognized and bound by TBP, which is a subunit of the TFIID complex in eu- karyotes, with RNA polymerases and associated factors. ${ }^{26,29)}$ Indeed, the deletion of TATA(Tal2) from the 5'-region was decreased the luciferase activity (Fig. 2B).

Therefore, to examine the transcriptional activity of TATA(Tal2), we utilized the two-hybrid system adapted for use in mammalian cells. ${ }^{30,31)}$ In this system, the association between one protein fused to the yeast GAL4 DNA-binding domain and the other protein fused to the herpes simplex virus VP16 activation domain promote the assembly of RNA polymerase II complexes at the TATA-box and increase the transcription of the firefly luciferase reporter gene. Two positive controls that encode Id fused to GAL4 (GAL4-Id) and MyoD fused to VP16 (VP16-MyoD) were contained in this system, and we utilized the vectors expressing these proteins to verify the transcriptional activity of TATA(Tal2). Moreover, we constructed the reporter vectors containing TATA(Tal2) instead of a minimal adenoviral promoter downstream of five GAL4 binding sites. These constructs were cotransfected into P19 cells, and the luciferase activity was measured. As a result, the luciferase activity from the reporter vector containing TATA(Tal2) was significantly increased in P19 cells that expressed both VP16-MyoD and GAL4-Id as compared with the reporter vector containing TATA(Ta12)Mut, including mutations in TATA(Tal2) (Fig. 2C). These results indicate 
A

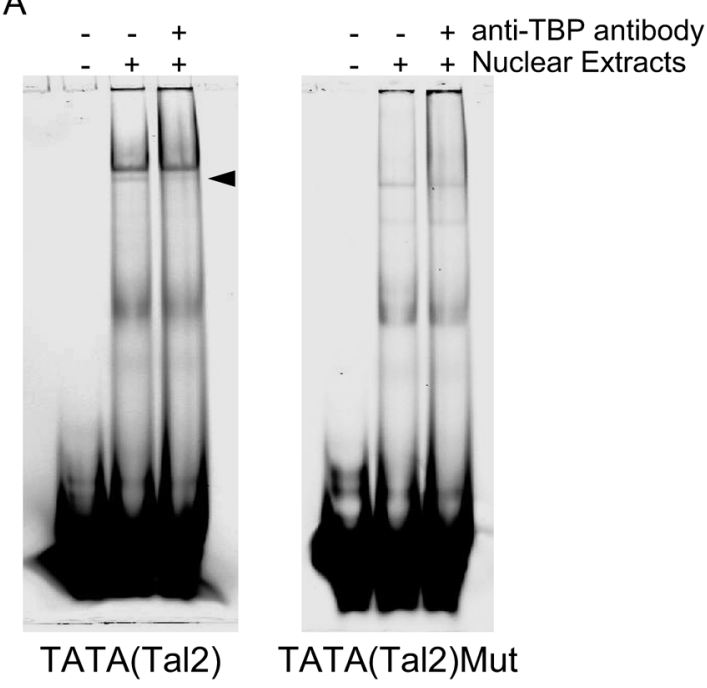

B

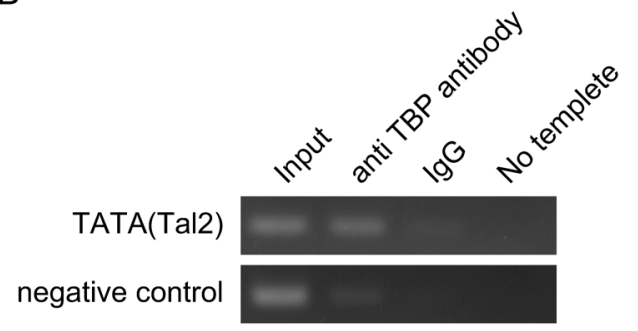

Fig. 3. Binding of TBP to TATA(Tal2)

(A) P19 cells were treated by atRA in suspension culture for $3 \mathrm{~h}$ and nuclear proteins were extracted from these cells. To verify the binding of TBP to TATA(Tal2), supershift analysis using anti-TBP antibody was performed. The disappearance of signals that indicated complexing with TBP and TATA(Tal2) was confirmed by analysis using Alexa 680-labeled TATA(Tal2) probes (black arrowhead). (B) The binding of TBP to the TATA-box-like element in P19 cells was verified by ChIP assay. Purified chromatin from P19 cells at $3 \mathrm{~h}$ after atRA treatment in suspension culture, which was immunoprecipitated with anti-TBP antibody or normal rabbit IgG, was subjected to PCR.

that TATA(Ta12) functions as a core promoter involved in transcription.

Next, to examine the binding of TBP to TATA(Tal2), we prepared the nuclear extracts from P19 cells treated by atRA in suspension culture and performed electrophoretic mobility shift assay (EMSA) using Alexa 680-labeled oligonucleotide probes containing TATA(Tal2) or TATA(Tal2)Mut. Although the pattern was different between these probes, several shifted complexes, which represented these probes bound to nuclear extracts, were detected. These complexes at $0 \mathrm{~h}$ were same as those at $3 \mathrm{~h}$ (data not shown). Therefore, binding of TBP to the Alexa 680-TATA(Ta2) probe was verified by supershift analysis of EMSA with the nuclear extract at $3 \mathrm{~h}$ (Fig. 3A). As a result, the disappearance of a shifted complex was confirmed by supershift analysis with anti-TBP antibody (black arrowhead in Fig. 3A), and it was confirmed that TBP binds to TATA(Tal2). Furthermore, we examined whether TBP binds to TATA(Tal2) in P19 cells by chromatin immunoprecipitation (ChIP) analysis (Fig. 3B). Chromatin from P19 cells $3 \mathrm{~h}$ after atRA treatment in suspension culture was immunoprecipitated with anti-TBP antibody or normal rabbit IgG. Then, binding DNA was purified and analyzed by PCR. PCR experiments showed that anti-TBP antibody, but not $\mathrm{IgG}$, immunoprecipitated the region containing TATA(Tal2), whereas negative controls were not immunoprecipitated with anti-TBP antibody. These results indicate that TBP binds to TATA(Tal2) in P19 cells after atRA treatment in suspension culture.

Transcription at the core promoter is mediated by basal transcription machinery that requires basal transcriptional factors. TFIID is one of these basal transcription factors, and the recognition of core promoter sequence motif is often performed by TFIID, which comprises TBP and TBP-associated factors (TAFs). ${ }^{27,28)}$ At least two distinct modes of transcription initiation are known: TATA-dependent initiation and CpG-island-based, TATA-independent initiation. ${ }^{26,29)}$ In this study, we found a TATA-box-like element "TATA(Tal2)" upstream of Tal2. TATA(Tal2) activated the transcription of genes similarly to the TATA-box in the core promoter, and TBP bound to TATA(Tal2) in P19 cells. Taken together, these results suggest that the regulation of Tal2 is TATA dependent, and that TATA(Tal2) participates in the transcription of Tal2.

Association between DR5(Tal2) and Tal2 Expression Tal2 is induced in P19 cells by atRA, which is involved in neural differentiation. ${ }^{5)}$ atRA signaling regulates the transcription of target genes through RARE. Thus, we searched for a RARE motif that might be involved in the transcription of Tal2, and found a putative motif, the RARE-like element "DR5(Tal2)." To examine the transcriptional activity of DR5(Tal2), we constructed reporter vectors containing three tandem repeats of DR5(Tal2) [DR5(Tal2) $\times 3]$, or DR5(Tal2)Mut [DR5(Tal2)Mut $\times 3$ ], including a mutation in DR5(Tal2), upstream of a minimal adenoviral promoter (Fig. 4A). In addition, we constructed an expression vector for RAR $\alpha$ cloned in-frame with the VP16 activation domain (VP16-RARA), which was constitutively active without atRA, ${ }^{32,33)}$ and measured the luciferase activities in P19 cells that were transfected with these vectors (Fig. 4B). The luciferase activity in P19 cells with both DR5(Tal2) $\times 3$ and VP16-RARA increased compared with the activity in P19 cells with DR5(Tal2)Mut $\times 3$ and VP16-RARA. Moreover, to examine whether atRA signaling activated transcription through DR5(Tal2), P19 cells that were transfected with these reporter vectors were treated with atRA in suspension culture, and then the luciferase activities in the cells were measured (Fig. 4C). Similar to the case of VP16-RARA, the luciferase activity in P19 cells with DR5(Tal2) $\times 3$ after atRA treatment in suspension culture increased compared with the activity in P19 cells with DR5(Tal2)Mut $\times 3$. These results indicate that transcription was activated through DR5(Tal2) in response to a constitutively active RAR $\alpha$ and atRA signaling in P19 cells.

Next, we prepared nuclear extracts from P19 cells at $0 \mathrm{~h}$ and $3 \mathrm{~h}$ after atRA treatment in suspension culture, and performed EMSA using these nuclear extracts and Alexa 680-labeled probes containing DR5(Tal2) or DR5(Tal2)Mut (Fig. 5A). A 


\section{A}

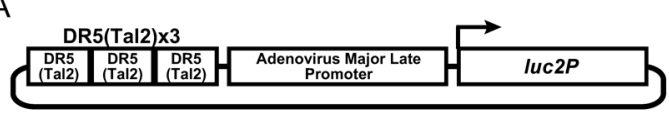

B

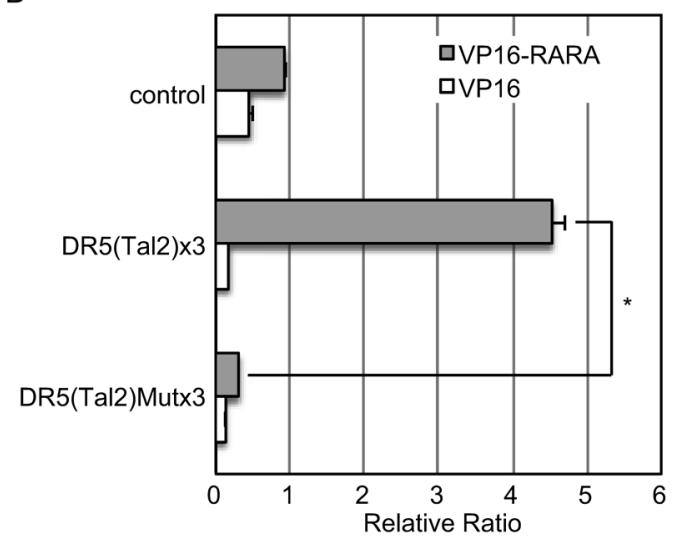

C

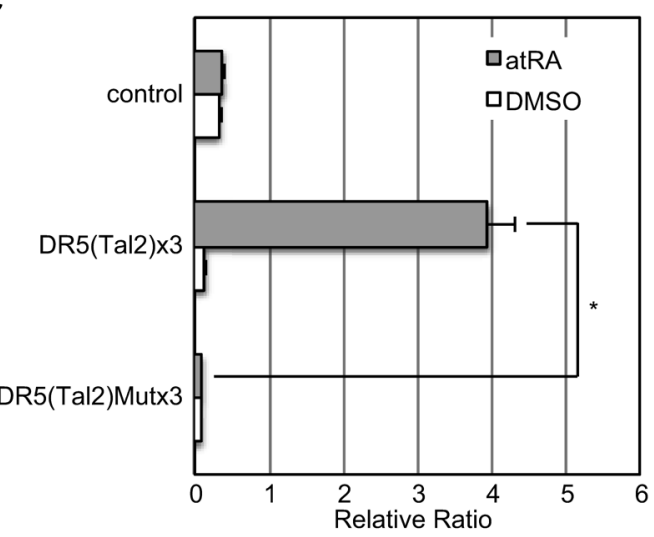

Fig. 4. Transcriptional Activity of DR5(Ta12) in P19 Cells

(A) The scheme of the constructed reporter vector. (B) P19 cells were cotransfected with the reporter vector containing DR5(Tal2) $\times 3$, DR5(Tal2)Mut $\times 3$ or GAL4binding domain as a control, and the vector expressed constitutive active RAR $\alpha$ (VP16-RARA). Luciferase activity was detected in P19 cells expressing VP16-RARA. Data represent means \pm S.E. of three independent experiments. $* p<0.05$. (C) Reporter vectors containing DR5(Tal2) $\times 3$ or DR5(Tal2)Mut $\times 3$ were transfected into P19 cells, followed by treatment with atRA in suspension culture. Luciferase activity was detected in these cells treated by atRA in suspension culture. As a control, reporter vector containing GAL4-binding domain was used. Data represent means \pm S.E. of three independent experiments. ${ }^{*} p<0.05$.

A

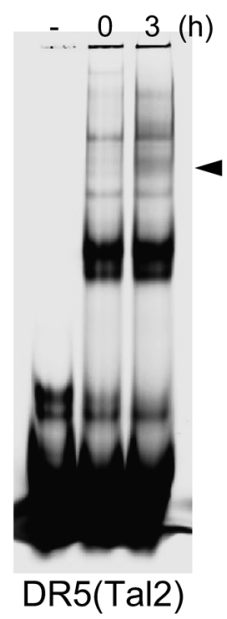

C

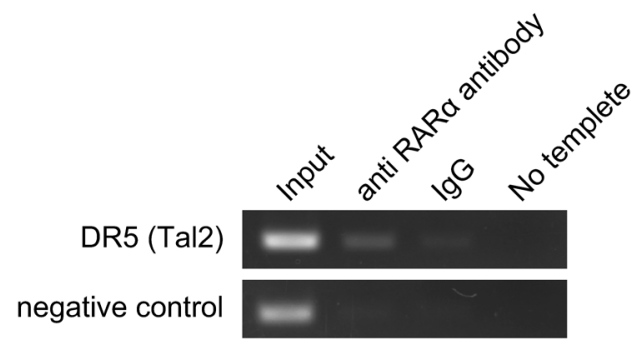

DR5(Tal2)Mut

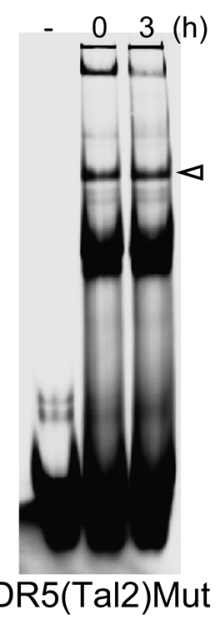

B

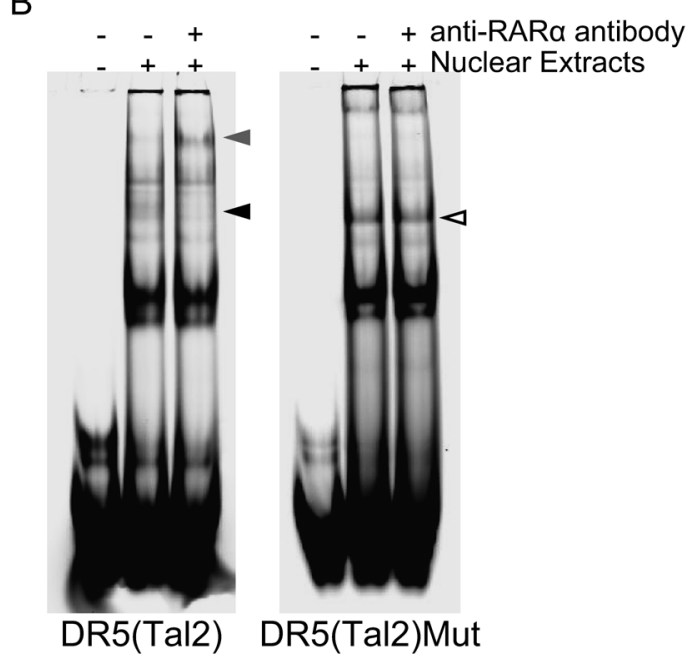

DR5(Tal2)Mut

Fig. 5. Binding of RAR $\alpha$ to DR5(Tal2)

(A) P19 cells were treated with atRA in suspension culture at $0 \mathrm{~h}$ and $3 \mathrm{~h}$. After extraction of nuclear protein from these cells, the binding of nuclear extracts to DR5(Ta12) was examined by EMSA using Alexa 680-labeled probes. A signal that indicated complexing with RAR $\alpha$ and DR5(Tal2) was detected (black arrowhead) in nuclear extracts at $3 \mathrm{~h}$. White arrowhead indicates the non-specific interaction. (B) To confirm the binding of RAR $\alpha$ to DR5(Tal2), supershift analysis using anti-RAR $\alpha$ antibody was performed. A shifted signal (gray arrowhead) that indicated complexing with RAR $\alpha$ and DR5(Tal2) was detected by the analysis using Alexa 680-labeled DR5(Ta12) probes. White arrowhead indicates the non-specific interaction. (C) Binding of RAR $\alpha$ to RARE-like element in P19 cells was verified by ChIP assay. Purified chromatin from P19 cells at $3 \mathrm{~h}$ after atRA treatment in suspension culture, which was immunoprecipitated with anti-RAR $\alpha$ antibody or normal rabbit IgG, was subjected to PCR. 
characteristic shifted complex with Alexa 680-DR5(Tal2) was detected in nuclear extracts at $3 \mathrm{~h}$ (black arrowhead in Fig. $5 \mathrm{~A})$ compared with $0 \mathrm{~h}$. This complex was not observed in the nuclear extracts with Alexa 680-DR5(Tal2)Mut. As RAR $\alpha$ is constantly expressed in P19 cells (Fig. 1C), this result may be correlated with the activation of Tal2 by atRA signaling. Moreover, to confirm the binding of RAR $\alpha$ to Alexa 680-DR5(Ta12), supershift analysis with anti-RAR $\alpha$ antibody was performed. Nuclear extracts at $3 \mathrm{~h}$ were used in this analysis. A specific shifted complex was detected in the nuclear extracts with Alexa 680-DR5(Tal2) and anti-RAR $\alpha$ antibody (gray arrowhead in Fig. 5B). The addition of anti-RAR $\alpha$ antibody did not change the mobility of any complexes including the complex (white arrowhead in Fig. 5) in the nuclear extracts with Alexa 680-DR(Tal2)Mut. Therefore, these results indicate that RAR $\alpha$ binds to DR5(Tal2). Subsequently, we examined by ChIP assay whether $\operatorname{RAR} \alpha$ bound to DR5(Tal2) in P19 cells (Fig. 5C). Similarly to anti-TBP antibody, chromatin from P19 cells at $3 \mathrm{~h}$ after atRA treatment in suspension culture was immunoprecipitated with anti-RAR $\alpha$ antibody, and purified binding DNA was analyzed by PCR. PCR experiments using specific primers showed that anti-RAR $\alpha$ antibody, but not $\mathrm{IgG}$, immunoprecipitated the region containing DR5(Tal2) in P19 cells, whereas negative controls were not immunoprecipitated with anti-RAR $\alpha$ antibody. Therefore, these results indicate that RAR $\alpha$ binds to DR5(Tal2) located in the intron of Tal2 in P19 cells.

Taken together, the RARE-like element "DR5(Tal2)" in the intron of Tal2, which consists of a direct repeat of the hexameric motif separated by $5 \mathrm{bp}$, responded to constitutively ac- tive RAR $\alpha$ and atRA signaling, and bound with RAR $\alpha$, which is a receptor for atRA, in P19 cells. These results suggest that DR5(Tal2) is involved in the transcription of Tal2 by atRA signaling in P19 cells.

Interaction between TATA(Tal2) and DR5(Tal2) In this paper, we showed that TATA(Tal2) in the 5'-region of Tal2 functioned as a core promoter and DR5(Tal2) in the intron of Tal2 responded to the atRA-RAR $\alpha$ signaling. It is known that RARs heterodimerize with retinoid X receptors (RXRs) and bind to RARE. In the absence of atRA, the heterodimer of RAR and RXR (RAR/RXR) is thought to constitutively bind to RARE and repress transcription through associations with the corepressors, nuclear receptor corepressor (NCoR) and silencing mediator for retinoid and thyroid hormone receptors (SMRT). Moreover, these corepressors interact with HDAC3. HDACs are able to deacetylate lysine residues of the N-terminal tails of histone and prevent transcription. Upon binding of atRA, RAR/RXR undergoes a conformational change resulting in the release of corepressor complexes, and associates with coactivators such as SRCs and $\mathrm{CBP} / \mathrm{p} 300$. The coactivators induce chromatin remodeling and facilitate the assembly of a transcription preinitiation complex containing TBP. ${ }^{11,34,35)}$ Therefore, it is anticipated that TATA(Tal2) and DR5(Tal2) interact through $\operatorname{RAR} \alpha$, coactivators and a transcription preinitiation complex containing TBP, and participate in the transcription of Tal2 in P19 cells (Fig. 6A).

We utilized ChIP assay to examine the interaction between TATA(Tal2) and DR5(Tal2) in P19 cells (Fig. 6B). In this assay, chromatin immunoprecipitated with anti-RAR $\alpha$ antibody was analyzed by PCR using specific primers for

A

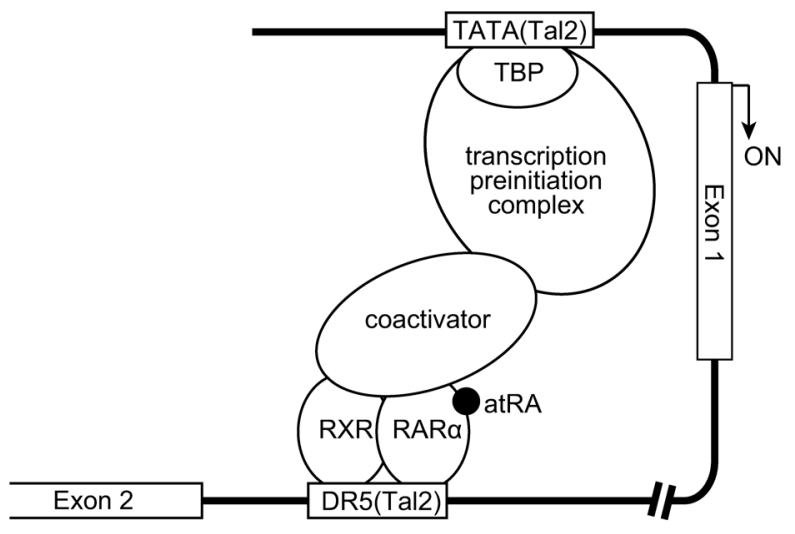

B

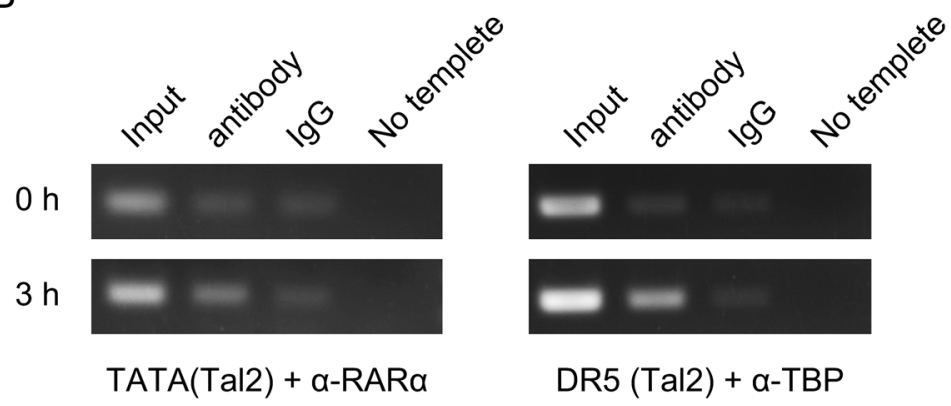

Fig. 6. Interaction between TATA(Tal2) and DR5(Tal2)

(A) The scheme of the transcriptional regulation of Tal2 that we anticipated. (B) The interaction between TATA(Tal2) and DR5(Tal2) was examined by ChIP assay. P19 cells were treated with atRA for $0 \mathrm{~h}$ or $3 \mathrm{~h}$ in suspension culture. Purified chromatin immunoprecipitated with anti-RAR $\alpha$ antibody was subjected to PCR using specific primers that were targeted to TATA(Tal2), and purified chromatin immunoprecipitated with anti-TBP antibody was subjected to PCR using specific primers that were targeted to DR5(Tal2). 
TATA(Ta12). Also, chromatin immunoprecipitated with antiTBP antibody was analyzed by PCR using specific primers for DR5(Tal2). Consequently, it was confirmed that RAR $\alpha$ was associated with TATA(Tal2), and that TBP was associated with DR5(Tal2). Therefore, our data suggested that DR5(Tal2) and TATA(Tal2) interacted through $\operatorname{RAR} \alpha$ and TBP in P19 cells, and support the possibility that TATA(Tal2) and DR5(Tal2) are regulatory elements which coordinately promote the transcription of Tal2 in P19 cells.

\section{CONCLUSION}

Because atRA signaling is important for neural differentiation, we believe that TAL2 induced by atRA commits target cells to the neural lineage. ${ }^{5}$ In this study, we focused on the regulatory region involved in the transcription of Tal2. Our data suggest that transcription of Tal2 is mediated by two distal regulatory elements: the TATA-box-like element "TATA(Tal2)" and the RARE-like element "DR5(Tal2)." There is a distance of approximately $5 \mathrm{~kb}$ between these elements. It is thought that TATA(Tal2) upstream of exon 1 functions as a core promoter, and that DR5(Ta12) located in the intron of Tal2 is bound with RAR $\alpha$ and responds to atRA signaling. To clarify the interaction between TATA(Tal2) and DR5(Tal2) in the transcription of Tal2, it is important to identify the cofactors, which are involved in the transcription of Tal2. Further work is underway to clarify the transcriptional regulation of Tal2 by atRA signaling in P19 cells.

Acknowledgments We would like to thank Yusuke Nakahira, Nana Okamoto, and Hiroaki Mori for technical support. This research was supported by a research grant from Tokushima Bunri University, Japan.

Conflict of Interest The authors declare no conflict of interest.

\section{REFERENCES}

1) Xia Y, Brown L, Yang CY, Tsan JT, Siciliano MJ, Espinosa R 3rd, Le Beau MM, Baer RJ. TAL2, a helix-loop-helix gene activated by the $(7 ; 9)(\mathrm{q} 34 ; \mathrm{q} 32)$ translocation in human T-cell leukemia. Proc. Natl. Acad. Sci. U.S.A., 88, 11416-11420 (1991).

2) Xia Y, Hwang LY, Cobb MH, Baer R. Products of the TAL2 oncogene in leukemic T cells: bHLH phosphoproteins with DNA-binding activity. Oncogene, 9, 1437-1446 (1994).

3) Mori S, Sugawara S, Kikuchi T, Tanji M, Narumi O, Stoykova A, Nishikawa SI, Yokota Y. The leukemic oncogene tal-2 is expressed in the developing mouse brain. Brain Res. Mol. Brain Res., 64, 199-210 (1999).

4) Bucher K, Sofroniew MV, Pannell R, Impey H, Smith AJ, Torres EM, Dunnett SB, Jin Y, Baer R, Rabbitts TH. The T cell oncogene Tal2 is necessary for normal development of the mouse brain. Dev. Biol., 227, 533-544 (2000).

5) Kobayashi $T$, Komori R, Ishida K, Kino K, Tanuma S, Miyazawa $\mathrm{H}$. Tal2 expression is induced by all-trans retinoic acid in P19 cells prior to acquisition of neural fate. Sci. Rep., 4, 4935 (2014).

6) Jones-Villeneuve EM, Rudnicki MA, Harris JF, McBurney MW. Retinoic acid-induced neural differentiation of embryonal carcinoma cells. Mol. Cell. Biol., 3, 2271-2279 (1983).

7) McBurney MW. P19 embryonal carcinoma cells. Int. J. Dev. Biol., 37, 135-140 (1993).

8) Bain G, Ray WJ, Yao M, Gottlieb DI. From embryonal carcinoma cells to neurons: the P19 pathway. BioEssays, 16, 343-348 (1994).

9) Maden M, Holder N. Retinoic acid and development of the central nervous system. BioEssays, 14, 431-438 (1992).

10) Maden M. Retinoic acid in the development, regeneration and maintenance of the nervous system. Nat. Rev. Neurosci., 8, 755-765 (2007).

11) Rhinn $M$, Dolle P. Retinoic acid signalling during development. Development, 139, 843-858 (2012).

12) Lu J, Tan L, Li P, Gao H, Fang B, Ye S, Geng Z, Zheng P, Song H. All-trans retinoic acid promotes neural lineage entry by pluripotent embryonic stem cells via multiple pathways. BMC Cell Biol., 10, 57 (2009).

13) $\overline{C h e n ~} \mathrm{Y}$, Reese DH. The retinol signaling pathway in mouse pluripotent P19 cells. J. Cell. Biochem., 112, 2865-2872 (2011).

14) Chambon P. A decade of molecular biology of retinoic acid receptors. FASEB J., 10, 940-954 (1996).

15) Balmer JE, Blomhoff R. Gene expression regulation by retinoic acid. J. Lipid Res., 43, 1773-1808 (2002).

16) Komori R, Kobayashi T, Matsuo H, Kino K, Miyazawa H. Csn3 gene is regulated by all-trans retinoic acid during neural differentiation in mouse P19 cells. PLoS ONE, 8, e61938 (2013).

17) McKenna NJ, O’Malley BW. Combinatorial control of gene expression by nuclear receptors and coregulators. Cell, 108, 465-474 (2002).

18) Cotnoir-White D, Laperriere D, Mader S. Evolution of the repertoire of nuclear receptor binding sites in genomes. Mol. Cell. Endocrinol., 334, 76-82 (2011).

19) Bastien J, Rochette-Egly C. Nuclear retinoid receptors and the transcription of retinoid-target genes. Gene, 328, 1-16 (2004).

20) Lalevée S, Anno YN, Chatagnon A, Samarut E, Poch O, Laudet V, Benoit G, Lecompte O, Rochette-Egly C. Genome-wide in silico identification of new conserved and functional retinoic acid receptor response elements (direct repeats separated by 5 bp). J. Biol. Chem., 286, 33322-33334 (2011)

21) Moutier E, Ye T, Choukrallah MA, Urban S, Osz J, Chatagnon A, Delacroix L, Langer D, Rochel N, Moras D, Benoit G, Davidson I. Retinoic acid receptors recognize the mouse genome through binding elements with diverse spacing and topology. J. Biol. Chem., 287, 26328-26341 (2012).

22) Gao X, Bian W, Yang J, Tang K, Kitani H, Atsumi T, Jing N. A role of $\mathrm{N}$-cadherin in neuronal differentiation of embryonic carcinoma P19 cells. Biochem. Biophys. Res. Commun., 284, 1098-1103 (2001).

23) Gaunt SJ, Paul YL. Origins of Cdx1 regulatory elements suggest roles in vertebrate evolution. Int. J. Dev. Biol., 55, 93-98 (2011).

24) Niwa H, Yamamura K, Miyazaki J. Efficient selection for highexpression transfectants with a novel eukaryotic vector. Gene, 108, 193-199 (1991)

25) National Center for Biotechnology Information. "Gene.": http:// www.ncbi.nlm.nih.gov/gene/21350), cited 26 August, 2014.

26) Ponjavic J, Lenhard B, Kai C, Kawai J, Carninci P, Hayashizaki Y, Sandelin A. Transcriptional and structural impact of TATAinitiation site spacing in mammalian core promoters. Genome Biol., 7, R78 (2006).

27) Juven-Gershon T, Hsu JY, Theisen JW, Kadonaga JT. The RNA polymerase II core promoter - the gateway to transcription. Curr. Opin. Cell Biol., 20, 253-259 (2008).

28) Kadonaga JT. Perspectives on the RNA polymerase II core promoter. Wiley Interdiscip. Rev. Dev. Biol., 1, 40-51 (2012).

29) Tora L, Timmers HT. The TATA box regulates TATA-binding protein (TBP) dynamics in vivo. Trends Biochem. Sci., 35, 309-314 (2010).

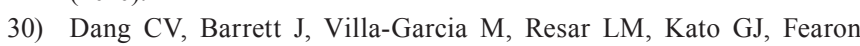
ER. Intracellular leucine zipper interactions suggest c-Myc heterooligomerization. Mol. Cell. Biol., 11, 954-962 (1991).

31) Fearon ER, Finkel T, Gillison ML, Kennedy SP, Casella JF, Tomaselli GF, Morrow JS, Van Dang C. Karyoplasmic interaction 
selection strategy: a general strategy to detect protein-protein interactions in mammalian cells. Proc. Natl. Acad. Sci. U.S.A., 89, 7958-7962 (1992).

32) Lipkin SM, Nelson CA, Glass CK, Rosenfeld MG. A negative retinoic acid response element in the rat oxytocin promoter restricts transcriptional stimulation by heterologous transactivation domains. Proc. Natl. Acad. Sci. U.S.A., 89, 1209-1213 (1992).

33) Lipkin SM, Grider TL, Heyman RA, Glass CK, Gage FH. Constitutive retinoid receptors expressed from adenovirus vectors that specifically activate chromosomal target genes required for differentia- tion of promyelocytic leukemia and teratocarcinoma cells. J. Virol., 70, 7182-7189 (1996).

34) Gillespie RF, Gudas LJ. Retinoid regulated association of transcriptional co-regulators and the polycomb group protein SUZ12 with the retinoic acid response elements of Hoxal, RARbeta(2), and Cyp26A1 in F9 embryonal carcinoma cells. J. Mol. Biol., 372, 298-316 (2007).

35) Al Tanoury Z, Piskunov A, Rochette-Egly C. Vitamin A and retinoid signaling: genomic and nongenomic effects. J. Lipid Res., 54, 1761-1775 (2013). 